\title{
Culture Methods to Study Apical-Specific Interactions using Intestinal Organoid Models
}

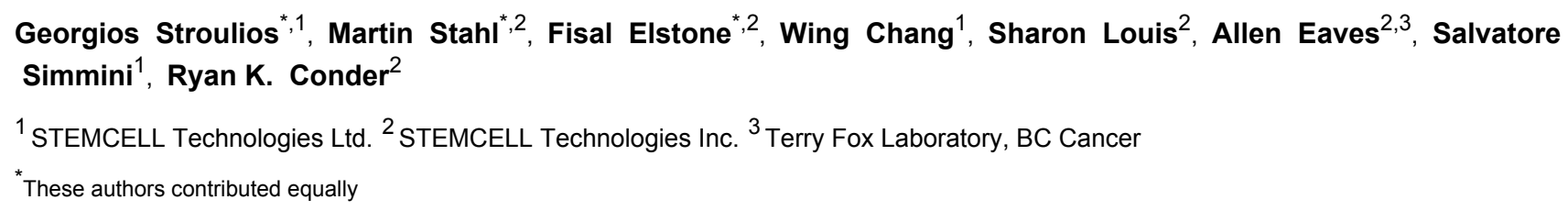

\section{Corresponding Authors}

Salvatore Simmini

salvatore.simmini@stemcell.com

Ryan K. Conder

ryan.conder@stemcell.com

\section{Citation}

Stroulios, G., Stahl, M., Elstone, F., Chang, W., Louis, S., Eaves, A., Simmini, S., Conder, R.K. Culture Methods to Study Apical-Specific Interactions using Intestinal Organoid Models. J. Vis. Exp. (169), e62330, doi:10.3791/62330 (2021).

\section{Date Published}

March 23, 2021

\section{DOI}

$10.3791 / 62330$

URL

jove.com/video/62330

\section{Abstract}

The lining of the gut epithelium is made up of a simple layer of specialized epithelial cells that expose their apical side to the lumen and respond to external cues. Recent optimization of in vitro culture conditions allows for the re-creation of the intestinal stem cell niche and the development of advanced 3-dimensional (3D) culture systems that recapitulate the cell composition and the organization of the epithelium. Intestinal organoids embedded in an extracellular matrix (ECM) can be maintained for long-term and self-organize to generate a well-defined, polarized epithelium that encompasses an internal lumen and an external exposed basal side. This restrictive nature of the intestinal organoids presents challenges in accessing the apical surface of the epithelium in vitro and limits the investigation of biological mechanisms such as nutrient uptake and host-microbiota/host-pathogen interactions. Here, we describe two methods that facilitate access to the apical side of the organoid epithelium and support the differentiation of specific intestinal cell types. First, we show how ECM removal induces an inversion of the epithelial cell polarity and allows for the generation of apical-out 3D organoids. Second, we describe how to generate 2-dimensional (2D) monolayers from single cell suspensions derived from intestinal organoids, comprised of mature and differentiated cell types. These techniques provide novel tools to study apical-specific interactions of the epithelium with external cues in vitro and promote the use of organoids as a platform to facilitate precision medicine.

\section{Introduction}

The intestinal epithelium is the second largest epithelium in the human body and consists of a polarized cell layer that facilitates nutrient uptake and acts as a barrier against environmental insults ${ }^{1}$. This distinction between the apical and basolateral sides allows cells of the epithelium to carry out their diverse functions. The apical compartment is exposed to the lumen and mediates the epithelial interactions with environmental stimuli and microorganisms, 
while also facilitating nutrient uptake. The basolateral surface houses intercellular junctions and cell-matrix adhesions, while interfacing with cells of the immune system and other tissues ${ }^{2}$. These junctions generate an impermeable monolayer attached to the basement membrane, which acts as a barrier and delivers the absorbed nutrients to the surrounding body tissue.

The establishment of culture systems that are able to recapitulate these intestinal functions in vitro has been challenging ${ }^{3}$. Conventional in vitro models utilize transformed human colorectal cancer cell lines, such as Caco-2, to generate 2D monolayer cultures. Despite being capable of modeling multiple functions of the absorptive compartment, these models cannot fully recapitulate the intestinal epithelium composition and function, which limit key functional characteristics and applications ${ }^{4,5}$.

The emergence of organoids as an advanced 3D culture system generated from stem cells that can self-organize and differentiate to organ-specific cell types, was a breakthrough in the in vitro study of the intestinal epithelium ${ }^{6}$. Intestinal organoids are embedded in an extracellular matrix (ECM) that resembles the basal lamina and form cell-matrix junctions that allow these cultures to retain the apicobasal polarity of the epithelium. Organoids exhibit an enclosed architecture in which the apical side is exposed to the luminal compartment, thus mimicking the structure of the intestine. Although this closed organization offers the opportunity to study orientationspecific functions, it limits investigations that require access to the apical side of the epithelium. Different approaches have been taken to overcome these limitations in both 2D and 3D, including organoid fragmentation, organoid microinjection, and generation of monolayer cultures ${ }^{7}$. Organoid fragmentation causes the loss of the structural organization and the destruction of cell junctions, which allows the exposure of the apical surface of the epithelium to the medium. This technique takes advantage of the regenerative capacity of the fragments to reform organoids when seeded into an extracellular matrix and has been used to model infectious disease and host-pathogen interactions ${ }^{8,9}$. However, the simultaneous access to both the apical and basal surface may also elicit non-specific responses to infection.

An alternative approach that allows access to the apical surface and preserves both the structural architecture and cell junctions is represented by the microinjection of factors into the lumen of organoids. This method has been extensively utilized to study host-pathogen interactions and model the effects of Cryptosporidium ${ }^{10}, H$. pylori ${ }^{11}$, and C. difficile ${ }^{12}$ on the gastrointestinal epithelium in vitro. Using similar techniques, the mutagenic potential of the $\mathrm{pks}^{+}$strain of $E$. coli on intestinal epithelium was determined ${ }^{13}$. Although effective, organoid microinjection is a laborious and inefficient task considering the high number of organoids that are needed to be injected to obtain measurable effects and therefore limits its application for high throughput assays.

Recent advances with intestinal organoids have also provided methods for the establishment of $2 \mathrm{D}$ monolayer organoid cultures, thereby exposing their apical surface $14,15,16,17$. These organoid-derived monolayers recapitulate key in vivo properties of the intestinal epithelium. They exhibit a physiologically relevant cell composition, containing both differentiated and stem cell populations and model the diversity across the crypt-villus axis. As apicobasal polarity is retained, the inherent monolayer properties allow for easy access of both the apical and basolateral sides and media exchanges can mimic intestinal flow and waste 
removal allowing for long-term culture. These features render organoid-derived monolayers amenable for studies focusing on luminal interactions and provide a superior model for epithelial barrier integrity and permeability ${ }^{18,19}$.

Studies have shown that epithelial cell polarity is tightly regulated by ECM proteins in MDCK spheroids 20,21 and recently in human intestinal organoids ${ }^{22}$. Removal of ECM components or inhibition of the integrin receptor that mediates the cell-matrix junctions results in a polarity reversal of intestinal organoids and the exposure of the apical side of the epithelium to the medium ${ }^{22}$. This approach has attracted the interest of researchers working on infectious diseases as it allows easy access to the apical side in 3D and makes it amenable to high throughput assays. Here, we describe a modified protocol based on the recent work by the Amieva $\mathrm{lab}^{22}$, that facilitates the generation of $3 \mathrm{D}$ intestinal organoids that readily expose their apical side. We also outline a protocol that can efficiently and reproducibly generate intestinal 2D monolayers derived from intestinal organoids.

\section{Protocol}

Derivation of human intestinal organoid cultures was performed as described elsewhere ${ }^{23}$. Organoids were maintained in culture as described by the Product Information Sheet (PIS) for the Intestinal Organoid Expansion Medium (refer to the Table of Materials).

\section{Inversion of intestinal organoid polarity}

NOTE: This section will outline the protocol for inverting the polarity of 3D intestinal organoids. This protocol provides a detailed procedure for the establishment of polarized organoids with an exposed apical surface in suspension in a culture plate. This protocol is intended as an end-point assay, although the organoids could be maintained in this conformation for over 2 weeks and maintain a small stem cell population that allows them to re-establish apical-in organoids upon passage.

1. Coating cultureware and tubes with anti-adherent solution

NOTE: To maximize the number of apical-out intestinal organoids and prevent undesirable attachment to the cultureware, precoating of cultureware is generally required. The section outlined below describes the coating of culture and plasticware with anti-adherent solution.

1. Coat culture plates to be used in the suspension part of the protocol as follows.

1. Add $0.5 \mathrm{~mL}$ of anti-adherent solution to each well of a 24-well tissue culture plate.

2. Swirl the plate to spread the solution evenly across the surface and the walls of the wells.

3. Centrifuge the plate at $1,300 \times g$ for $10 \mathrm{~min}$.

4. Remove anti-adherent solution from each well using an aspirator or a $1 \mathrm{~mL}$ pipette.

5. Wash the wells with $1 \mathrm{~mL}$ of $\mathrm{DMEM} / \mathrm{F}-12$ with 15mM HEPES (DMEM/F12).

6. Fill each washed well with $0.5 \mathrm{~mL}$ of DMEM/ $\mathrm{F}-12$ and store the plate at $37^{\circ} \mathrm{C}$ and $5 \% \mathrm{CO}_{2}$ until use.

NOTE: If not used immediately, the coated plates can be kept in the incubator for at least 1 week.

2. Coat $15 \mathrm{~mL}$ conical tubes used in this protocol as follows. 
1. Add $4 \mathrm{~mL}$ of anti-adherent solution to the $15 \mathrm{~mL}$ conical tube.

2. Swirl the tube to spread the solution evenly across the surface of the walls.

3. Centrifuge the tube at $1,300 \times g$ for $10 \mathrm{~min}$.

4. Remove the anti-adherent solution from the tube.

5. Wash the tube $1 \mathrm{x}$ with $5 \mathrm{~mL}$ of DMEM/F-12 and aspirate the DMEM/F-12. The tubes are now ready for use.

NOTE: If not used immediately, add $5 \mathrm{ml}$ of DMEM/F12 and store at $4{ }^{\circ} \mathrm{C}$. Coated tubes can be kept at $4{ }^{\circ} \mathrm{C}$ for at least a week.

2. Polarity inversion of intestinal organoids by suspension culture

NOTE: The steps below outline the inversion of intestinal organoids previously cultured under standard ECM dome conditions. The procedures described here are for one well of a 24-well plate. If using other cultureware, adjust volumes accordingly.

1. Carefully remove and discard the medium from each well containing organoids without disrupting the basement membrane extracellular matrix dome. Ensure that the size of the organoids is of $150-250$ $\mu \mathrm{m}$ diameter (typically at day 3-5) prior to beginning the inversion protocol.

2. Add $1 \mathrm{~mL}$ of ice-cold dissociation solution in each well.

3. Incubate at room temperature $\left(15-25^{\circ} \mathrm{C}\right)$ for $1 \mathrm{~min}$.

4. Add at least $2 \mathrm{~mL}$ of anti-adherent solution to a 15 $\mathrm{mL}$ tube to be used for coating plastic tips.
5. Add at least $2 \mathrm{~mL}$ of $\mathrm{DMEM} / \mathrm{F}-12$ to a $15 \mathrm{~mL}$ tube to be used for washing plastic tips rinsed with antiadherent solution.

6. Coat a $1 \mathrm{~mL}$ pipette tip with anti-adherent solution, pipetting $1 \mathrm{~mL}$ of solution three times into the tube with anti-adherent solution from step 1.2.4.

7. Wash the tip in cold DMEM/F-12, pipetting $1 \mathrm{~mL}$ of solution three times into the tube with DMEM/F-12 from step 1.2.5.

8. Using the coated tip, carefully dislodge the domes by pipetting slowly. Take care not to disrupt or fragment the organoids.

9. Transfer the organoid suspension to a plate treated with anti-adherent solution (from step 1.1.1).

10. Place the plate on a shaker at $4{ }^{\circ} \mathrm{C}$ for $30 \mathrm{~min}$. A gyro shaker can be used at $70 \mathrm{rpm}$.

NOTE: Avoid harsh shaking, such as vortexing the sample, as it can result in organoid fragmentation. The formation of bubbles and froth can indicate harsh shaking.

11. After $30 \mathrm{~min}$, remove the plate. Using a $1 \mathrm{~mL}$ pipette tip coated with anti-adherent solution, gently pipette the solution up and down.

12. Place the plate on a shaker at $4{ }^{\circ} \mathrm{C}$ for $15 \mathrm{~min}$. A gyro shaker can be used at $70 \mathrm{rpm}$.

13. Remove the plate and let the organoids settle by gravity (1-2 $\mathrm{min}$ at room temperature). Observe the plate under the microscope to verify organoid sedimentation.

NOTE: Avoid extensive agitation of the plate as it can cause settled organoids to resuspend. 
14. After the organoids settle, remove as much of the dissociation solution as possible and wash by adding $1.5 \mathrm{~mL}$ of DMEM/F-12.

15. Allow the organoids to sediment and remove as much of the supernatant as possible. Repeat the wash step once more.

NOTE: Observe the plate under the microscope to verify organoid sedimentation before performing any washing step.

16. Remove as much of the $\mathrm{DMEM} / \mathrm{F}-12$ as possible and add $0.5 \mathrm{~mL}$ of Intestinal Organoid Expansion Medium. Incubate at $37{ }^{\circ} \mathrm{C}$ and $5 \% \mathrm{CO}_{2}$.

17. On the following day, perform a partial medium change by tilting the plate on a 25 - to 30 -degree angle, and removing medium along the wall of the well. Remove $0.4 \mathrm{~mL}$ of medium taking care not to remove suspended organoids.

18. Add $0.4 \mathrm{~mL}$ Intestinal Organoid Expansion medium. Incubate at $37^{\circ} \mathrm{C}$ and $5 \% \mathrm{CO}_{2}$ for 3 days.

NOTE: After 3 days, most of the organoids should have an apical-out polarity, but large aggregates may have formed.

19. If aggregates have formed, use a $1 \mathrm{~mL}$ pipette with a tip coated with anti-adherent solution (as described in steps 1.2.6 and 1.2.7) to shear the aggregates by pipetting up and down 20 times while pressing the end of the tip into the bottom of the plate.

20. Place the plate at $37^{\circ} \mathrm{C}$ and $5 \% \mathrm{CO}_{2}$ and perform a full medium change the next day with Intestinal Organoid Expansion medium (as described in section 1.2.17).
21. After 2 days (day 5 in suspension), apical-out intestinal organoids can be used in downstream assays.

\section{Establishment of intestinal cell 2D monolayer and Air-Liquid Interface (ALI) cultures derived from 3D intestinal organoids}

NOTE: This section will outline the protocol for generating 2D monolayer cultures from intestinal organoids. This technique provides the advantage of establishing a confluent, polarized monolayer culture with an exposed apical surface in a tissue culture plate containing cell culture membrane insert. Although the monolayer will begin to differentiate in the submerged, monolayer format, additional differentiation of the monolayer can be achieved by switching to an ALI culture after it has reached confluence. Both the monolayer and subsequent $\mathrm{ALI}$ protocols are intended as end-point assays and although the monolayer culture maintains a small stem cell population, neither can be efficiently split and passaged after it is established.

1. Preparing media and plates for intestinal monolayer culture

1. Prepare Intestinal Organoid Differentiation Medium as outlined by the manufacturer for monolayer culture (see List of Materials). Add Y-27632 only to a volume of medium that will be used within 1 week at a final concentration of $10 \mu \mathrm{M}$. If not used immediately, store at $4{ }^{\circ} \mathrm{C}$.

2. Pre-warm Trypsin EDTA (0.05\%) to $37^{\circ} \mathrm{C}$.

3. At least $2 \mathrm{~h}$ prior to seeding monolayer cultures, prepare a $2 \%$ ECM coating solution as follows.

1. Thaw ECM on ice and add 1:50 to cold, sterile PBS to prepare a $2 \%$ solution. Prepare 
sufficient quantities to add $100 \mu \mathrm{L}$ to the upper well of each $6.5 \mathrm{~mm}\left(0.33 \mathrm{~cm}^{2}\right)$ cell culture membrane insert to be used. Adjust the volume appropriately for larger or smaller cultures.

4. Add $100 \mu \mathrm{L}$ to each well and incubate each plate at $37^{\circ} \mathrm{C}$ and $5 \% \mathrm{CO}_{2}$ until needed (at least $2 \mathrm{~h}$ prior to seeding).

2. Dissociating intestinal organoids for monolayer generation and culture

1. Remove an appropriate number of organoid culture wells from the incubator. Refer to Table 1 for the recommended number of wells to harvest for various cultureware.

2. Aspirate all medium from the organoid cultures without disturbing the ECM dome.

3. Add $1 \mathrm{~mL}$ of dissociation solution to each well.

4. Incubate at room temperature $\left(15-25^{\circ} \mathrm{C}\right)$ for at least $1 \mathrm{~min}$.

5. Using a $1 \mathrm{~mL}$ pipette, vigorously pipette up and down to disrupt the dome and release the organoids.

6. Pool the suspended organoids from each well in a $15 \mathrm{~mL}$ conical tube. Incubate at room temperature for 10 min with gentle agitation or rocking. Organoids for seeding multiple wells can be pooled at this step, up to $10 \mathrm{~mL}$ volume in each tube.

7. Centrifuge at $200 \times g$ for $5 \mathrm{~min}$ at $4{ }^{\circ} \mathrm{C}$.

8. Remove and discard the supernatant. Add $5 \mathrm{~mL}$ icecold DMEM/F-12 to resuspend organoids.

9. Mix and centrifuge again at $200 \times g$ for 5 min at $4{ }^{\circ} \mathrm{C}$.

10. Aspirate the supernatant, removing as much as possible, being careful not to disturb the pellet.
NOTE: Some residual ECM may remain in the pellet; however, this should not significantly affect the dissociation of the organoids.

11. Add $1 \mathrm{~mL}$ of pre-warmed $\left(37^{\circ} \mathrm{C}\right)$ Trypsin-EDTA $(0.05 \%)$ to resuspend organoids. Mix thoroughly to ensure an even suspension. Add up to an additional $1 \mathrm{~mL}$ of Trypsin-EDTA for a large number of cells, or if a significant amount of ECM remains.

12. Incubate at $37^{\circ} \mathrm{C}$ for $5-10 \mathrm{~min}$.

13. Mix thoroughly with a $1 \mathrm{~mL}$ pipette to disrupt the organoids as much as possible. Organoids should be completely dissociated into single cells or small fragments. If larger fragments or whole organoids remain after thoroughly pipetting, continue the incubation with Trypsin-EDTA at $37^{\circ} \mathrm{C}$ for another 3-5 $\min$.

NOTE: Do not incubate with Trypsin-EDTA for more than $20 \mathrm{~min}$, as this may result in an increased loss of cell viability.

14. Once organoids are sufficiently dissociated, add an equal volume of DMEM/F-12 (e.g., $1 \mathrm{~mL}$ DMEM/ F-12 per $\mathrm{mL}$ Trypsin-EDTA) and pipette up and down to mix thoroughly. Inactivate Trypsin-EDTA by adding $10 \%$ FBS to the DMEM/F-12 in this step.

15. Centrifuge fragments at $200 \times g$ for $5 \mathrm{~min}$ at $2-8^{\circ} \mathrm{C}$.

16. If the dissociated organoids fail to pellet, this is common and may be due to an accumulation of mucus released by the dissociated cells. In this case, mix the cells thoroughly by pipetting up and down and centrifuge them again at $200 \times g$ for $5 \mathrm{~min}$ at 2-8 ${ }^{\circ} \mathrm{C}$.

17. Carefully remove as much supernatant as possible, leaving only the cell pellet. 
18. Resuspend the cells in $100 \mu \mathrm{L}$ of Intestinal Organoid Differentiation medium (with $10 \mu \mathrm{M} \mathrm{Y}$-27632) for each well to be seeded. Adjust the volume appropriately for larger or smaller well sizes.

19. Remove the coated plates from the incubator (prepared in step 3.1.4) and remove the excess ECM from each well.

20. Add $100 \mu \mathrm{L}$ of the cell suspension to the upper well of each cell culture insert.

21. Add $500 \mu \mathrm{L}$ of Intestinal Organoid Differentiation medium to the lower well of each cell culture insert.

22. Incubate at $37^{\circ} \mathrm{C}$ and $5 \% \mathrm{CO} 2$.

23. Replace the medium in both the upper and lower wells every 2 to 3 days. Monolayer cultures should reach confluency within 7 days and will often reach confluency within 2-3 days.

3. Establishing an Air-Liquid Interface (ALI) culture NOTE: If desired, further differentiation of a submerged intestinal monolayer culture can be accomplished by transitioning the submerged monolayer culture to an ALI culture. This culture method will allow an increase in the number of differentiated cell types, particularly cells of the secretory lineage, such as goblet and enteroendocrine cells.

1. Establish a monolayer culture in a cell culture insert as described above in steps 2.2.1-2.2.23 and maintain this culture at $100 \%$ confluency for at least 4 days.

2. To establish an ALI culture, remove medium from the upper and lower wells. Add fresh Intestinal Organoid Differentiation medium (with Y-27632) to the lower well, leaving the upper well empty.
3. Incubate at $37^{\circ} \mathrm{C}$ and $5 \% \mathrm{CO}_{2}$.

4. Replace the medium in the lower well every $2-3$ days and allow the monolayer to differentiate for at least 1 week.

5. Rinse the upper well with sterile PBS to remove excess mucus accumulation, if needed.

Under these conditions, the ALI culture can be maintained for at least 2-3 weeks.

\section{Representative Results}

Organoids were generated from biopsy samples following the protocol described previously ${ }^{23}$ and in the PIS for Intestinal Organoid Expansion Medium (see the Table of Materials). Figure 1A, Left Panel, shows the phenotype of the intestinal organoids cultured in a dome with Intestinal Organoid Expansion Medium. In these culture conditions, organoids exhibit a cystic morphology defined by a thin (10-25 $\mu \mathrm{m})$ epithelium that encloses a lumen (Figure 1A, Right Panel). At this stage, the apical side of the intestinal epithelium faces the lumen, while the basolateral side contacts the surrounding extracellular matrix. When the majority of the organoids reached the desired size, the extracellular matrix was removed, and the organoids were then cultured in suspension. The loss of cellular binding to the extracellular matrix triggers an inversion process in the organoids, resulting in a reversal of the polarity of the organoid epithelium, exposing the apical side of the epithelium to the growth medium and internalizing the basolateral side.

In some cultures, organoids in suspension aggregate and fuse, an effect which is more profound during the first 3 days (Figure 1B, Left Panel). Application of a shearing technique allows detachment of the organoids and continuation of the 
cultures for days with minimal reaggregation (Figure 1B, Right Panel).

Intestinal organoids cultured in ECM domes continue to expand (Figure 1C, Left Panel) and exhibit spontaneous formation of secondary budding structures, resembling small crypts, on the basolateral side of the epithelium surrounding the lumen (Figure 1C, Right Panel). At the same time point, organoids maintained for 5 days in the absence of extracellular matrix continue to develop in suspension (Figure 1D, Left Panel). Inversion of the polarity is characterized by the thickening $(30-40 \mu \mathrm{m})$ of the epithelium that surrounds the core of the organoids and the appearance of a variety of morphologies: elongated (Figure 1D, Right Panel and Supplementary Figure 1A), cystic (Supplementary Figure 1B), and irregular (Supplementary Figure 1C). This is often combined with a shrinking of the luminal space within the organoid, impacting their overall size.

Efficient inversion can also be confirmed by analyzing the expression of intestinal-specific polarity markers. Apical-out intestinal organoids show a distinct localization of the nuclei toward the lumen of the organoid, as indicated by the 4',6diamidino-2-phenylindole (DAPI) signal. The expression of apical markers, such as VILLIN (Figure 2A) and ZO-1 (Figure 2B), is detected at the outer side of the epithelium that is exposed to the medium. This localization is in stark contrast with that observed in intestinal organoids cultured in ECM. Extracellular matrix embedded organoids stained for nuclei (DAPI), VILLIN (Figure 2C), and ZO-1 (Figure 2D) demonstrate an apicobasal polarity where the apical side is facing the lumen of the organoid.

Complete removal of ECM is required to obtain efficient polarity inversion of the intestinal organoids. Occasionally, a portion of organoids found in suspension cultures surrounded by ECM residues, shows a cystic morphology that suggests a failure in polarity inversion of the epithelium (Supplementary Figure 2A). Analysis of immunofluorescent staining, performed on these organoids, provides evidence of the basolateral position of the nuclei (DAPI) along the epithelium and the expression of ZO-1 at the apical side that faces the lumen of the organoid (Supplementary Figure 2B) confirming that incomplete ECM removal causes retention of the apicobasal polarity in a manner similar to ECM-embedded organoids.

The protocol for the establishment of intestinal monolayer cultures results in a confluent monolayer culture within 7 days of seeding and the culture will often reach confluence within as little as 2-3 days. One of the major determinants of success is the number and quality of cells used to seed the monolayer. Figure 3A, Left Panel provides an example of an ideal seeding density of approximately 150,000 cells in a 6.5 $\mathrm{mm}$ cell culture membrane insert. This number is not fixed and can be highly variable based on the donor and the quality of the source organoid culture; therefore, the cell number should be optimized based on these variables. If the seeding density is too low, or of poor quality (Figure 3A, Right Panel), there may not be sufficient attachments to form a confluent monolayer culture.

Once the monolayer is established (Figure 3B), the cells form tight junctions, creating a cobblestone appearance (Figure 3B, Left Panel). If they fail to form a confluent monolayer (Figure 3B, Right Panel), the appearance of the monolayer will often be "patchy", with regions of good quality cell attachment, but with larger gaps between these regions. These cultures do not provide a functional barrier between the basal and apical compartments and are not suitable for the described assays. A confluent monolayer 
orients its VILLIN-containing brush border toward the apical side of the epithelium, with its nucleus positioned toward the basolateral pole of the cell (Figure 4B). In between cells, intercellular junctions consisting of multi-protein complexes, including ZO-1 are formed (Figure 4B). Their presence is key to providing the barrier function of the epithelial culture.

Once confluent, the transition to an ALI culture induces further differentiation of the culture (Figure 3C). Small, round goblet cells appear and the monolayer itself takes on a more folded appearance. Although goblet cells are present within the epithelium of submerged culture (Figure 4A), they are more prominent following ALI differentiation. Goblet cells present within the epithelium secrete mucus, leading to a hazy appearance over the top of the epithelium. The goblet cells and secreted mucus can be visualized by staining for the secreted mucin protein, MUC2 (Figure 4A, C and D) and the increase in the goblet cell population can be measured by an increase in MUC2 expression (Supplementary Figure 3A). It is not necessary to remove this gel-like mucus layer and it will stick to the surface of the epithelium and remain following repeated washes. If removal is necessary, washing the culture with a mucolytic compound, such as $10 \mathrm{mM} \mathrm{N}$ acetyl cysteine or $50 \mu \mathrm{g} / \mathrm{mL}$ DTT removes excess mucus. In addition to the increase in the goblet cell population, the ALI interface also increases the presence of enteroendocrine cells (as indicated by CHGA expression) (Supplementary Figure 3B) and mature enterocytes (as indicated by KRT20 expression) (Supplementary Figure $3 \mathbf{C}$ ).
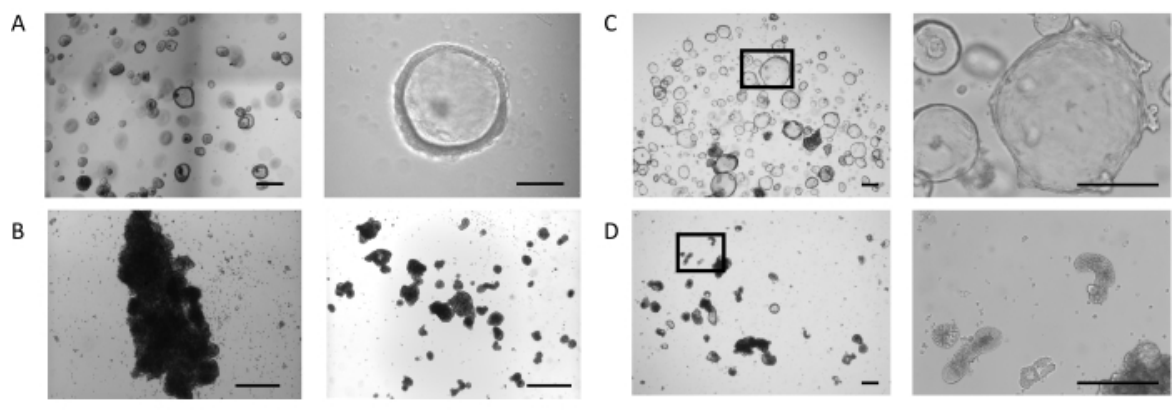

Figure 1: Stages of the apical-out intestinal organoid generation. (A) Representative images of a dome with organoids of the desired size at day 4 (Left Panel, Scale bar $=500 \mu \mathrm{m}$ ). Organoids are thin walled, with an open luminal compartment (Right Panel, Scale bar $=100 \mu \mathrm{m}$ ). (B) Representative image of a well with extensive aggregation after 3 days in suspension (Left Panel, Scale bar $=200 \mu \mathrm{m})$. Image of clump fragments directly after shearing (Right Panel, Scale bar $=200 \mu \mathrm{m}) .(\mathrm{C})$ Representative image of intestinal organoids in dome at day 7. Organoids display an expanded lumen with the formation of small buds on the basolateral side of the epithelium (Left 20x magnification, Right 100x magnification of the marked region, Scale bar $=200 \mu \mathrm{m}$ ). (D) Representative image of intestinal organoids after ECM removal and subsequent suspension culture for 5 days. The organoids obtain a dense morphology with a thickened epithelium and expose their apical side to the medium. (Left 20x magnification, Right 100x magnification of the marked region, Scale bar $=200 \mu m$ ). Please click here to view a larger version of this figure. 
A

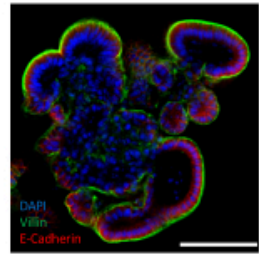

B

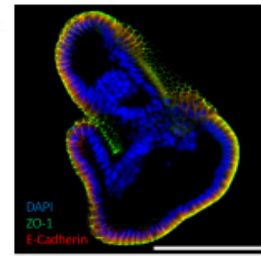

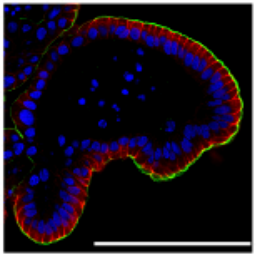

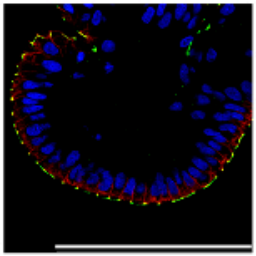

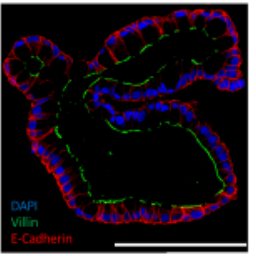

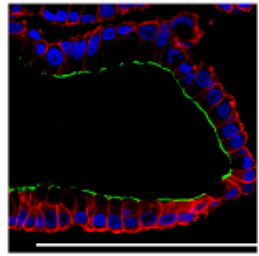

D
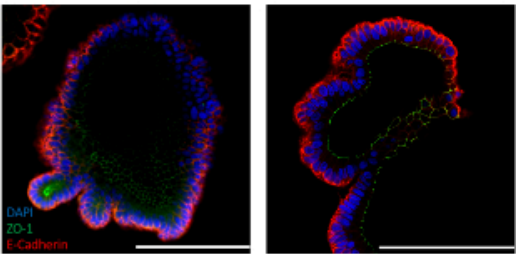

Figure 2: Immunofluorescent staining for cell polarity markers in intestinal organoids. Apical-out (A,B), and apicalin (C,D) oriented intestinal organoids were stained with apical markers ZO-1 and VILLIN, and with epithelial marker ECADHERIN (red). DAPI (blue) was used to visualize nuclei. Left panels display images taken at 25x magnification and right panels display images of different organoids at $63 x$ magnification (only panel $\mathrm{C}$ displays $25 \mathrm{x}$ and $63 \mathrm{x}$ magnification of the same organoid). (A) Apical-out intestinal organoids stained with VILLIN (green) and E-CADHERIN (red) indicate the exposure of the apical side to the medium. (B) Apical-out intestinal organoids stained with ZO-1 (green) and E-CADHERIN (red) show the presence of tight junctions and reversion of the apicobasal polarity. (C) Matrigel-embedded intestinal organoid stained with VILLIN (green) and E-CADHERIN (red) showing the apical side facing the organoid lumen. (D) Matrigelembedded intestinal organoids stained with ZO-1 (green) and E-CADHERIN (red) indicating the presence of apical tight junctions facing the lumen of the organoid. (Scale bar $=100 \mu \mathrm{m}$ ). Organoids were stained by immunofluorescence and imaged using previously published protocols ${ }^{24,25}$. Please click here to view a larger version of this figure. 
A
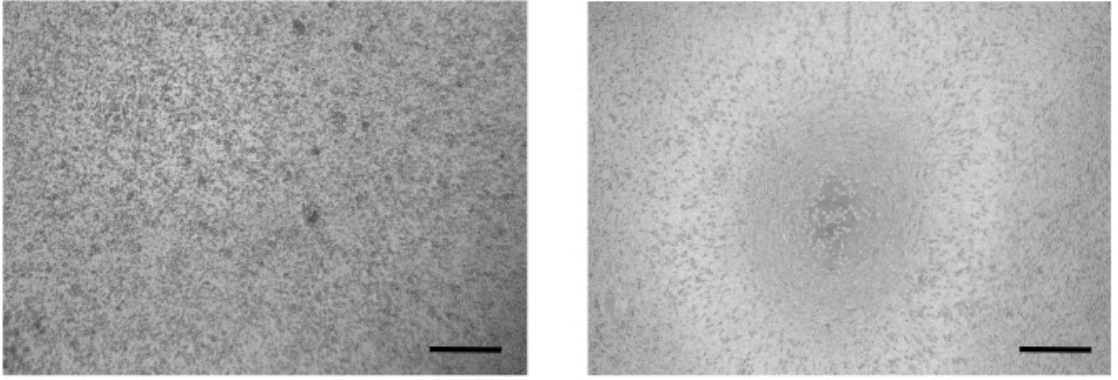

B
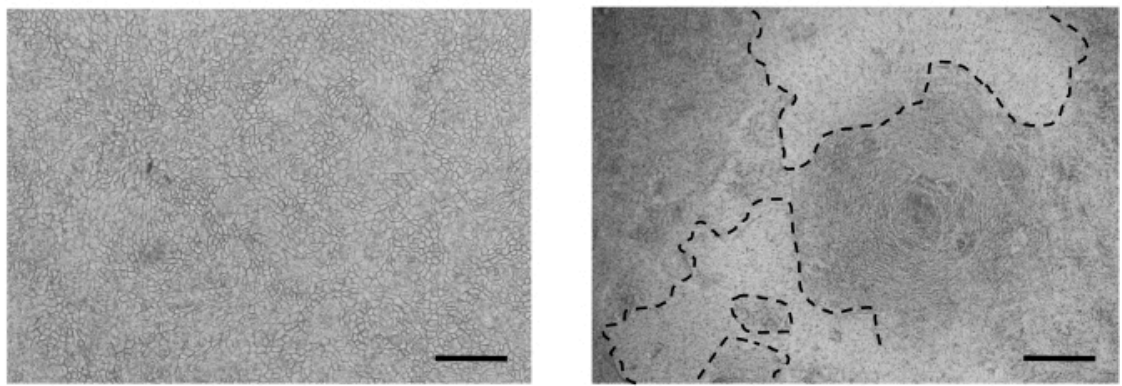

C

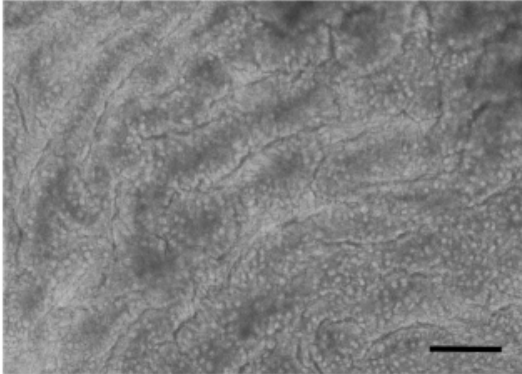

Figure 3: Establishment of intestinal monolayer cultures. (A) Representative image of 3D organoids following treatment with $0.05 \%$ Trypsin-EDTA. Organoids are dissociated to single cells or small cell-clumps in preparation for seeding monolayer cultures. Left Panel: example of an optimal seeding density for a monolayer culture, approximately 150,000 cells per $100 \mu \mathrm{L}$ on a $6.5 \mathrm{~mm}$ cell culture membrane insert. Right Panel: example of suboptimal seeding density at $<50,000$ cells per $100 \mu \mathrm{L}$ on a $6.5 \mathrm{~mm}$ cell culture membrane insert. (B) Representative brightfield image of a submerged monolayer culture. Left Panel: 100\% confluent layer with the characteristic cobblestone appearance. Right Panel: approximately $50 \%$ confluent monolayer. Gaps seen in the monolayer (indicated by dashed line) close over time due to the continued proliferation of the intestinal stem cells. (C) Representative brightfield image of a differentiated ALI culture at 7 days. (Scale bar $=200 \mu \mathrm{m})$. Please click here to view a larger version of this figure. 
A

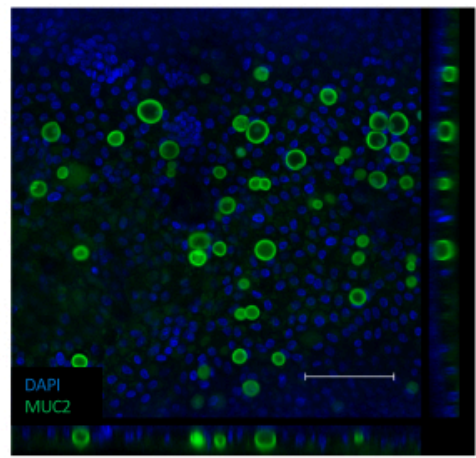

B

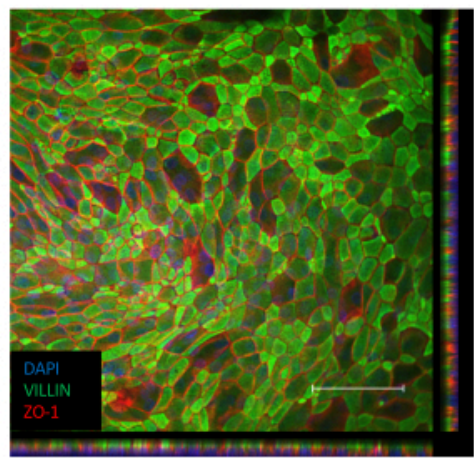

C

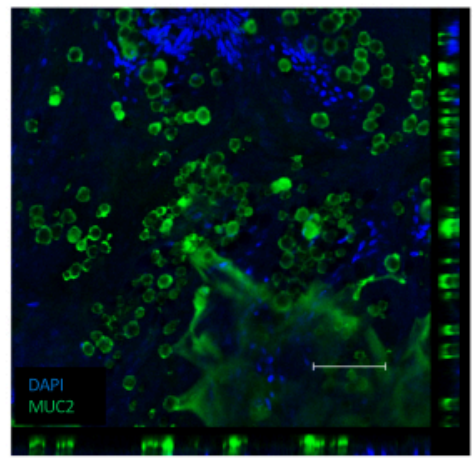

D

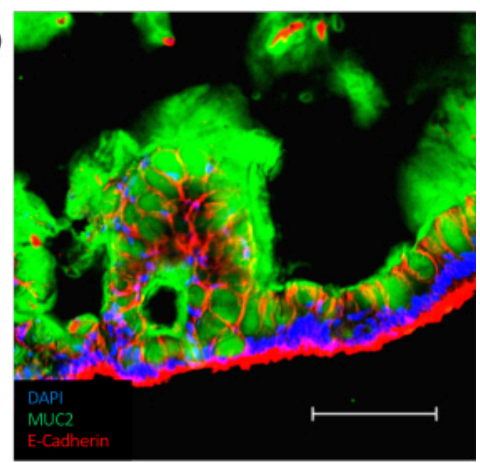

Figure 4: Immunofluorescent staining for differentiated cell markers in monolayer cultures. (A) Z-stack image of immunofluorescent staining of a submerged monolayer culture for the mucin protein MUC2, indicating the presence of goblet cells within the monolayer culture (green = MUC2, blue = DAPI). (B) Z-stack image of immunofluorescent staining of a submerged monolayer. VILLIN staining (green) along the apical end of the epithelium indicates the presence of a brush border and ZO-1 staining (red) indicates the presence of tight junctions between cells (blue = DAPI). (C) Z-stack image of immunofluorescent staining of an ALI-differentiated monolayer culture for the mucin protein MUC2, indicating the presence of a significantly larger number of goblet cells within the ALI monolayer culture (green = MUC2, blue = DAPI). (D) Cryosection of the ALI-differentiated monolayer culture, stained for the presence of MUC2 (green) and E-CADHERIN (red) indicating the presence of goblet cells in the epithelium and the secretion of mucus along the apical side of the monolayer culture. $($ Scale bar $=200 \mu \mathrm{m})$. Monolayer cultures were stained by immunofluorescence and imaged using previously published protocols ${ }^{26,27}$. Please click here to view a larger version of this figure.

\section{Supplementary Figure 1: Spectrum of phenotypes of} apical-out intestinal organoid in culture suspension. $(\mathbf{A}, \mathbf{B}, \mathbf{C})$ Additional representative images of intestinal organoid morphologies maintained in suspension 5 days after ECM removal. Organoid polarity has inverted. The organoids have become more dense with a thickened epithelium and the apical side of the organoids is facing outward. Organoids can show a variety of morphologies: elongated (A), cystic (B), and irregular $(\mathbf{C})$. (Scale bar $=100 \mu \mathrm{m})$. Please click here to download this File. 


\section{Supplementary Figure 2: Intestinal organoids fail to} invert polarity in the presence of residual basement membrane matrix medium in suspension cultures. (A) Representative image of incomplete ECM removal and failure to invert polarity of the organoids. Matrigel remnants are present around the organoids and contribute in maintaining the epithelium polarity oriented apical-in. Organoids show a cystic morphology with a thin epithelium surrounding the lumen $($ Scale bar $=200 \mu \mathrm{m})$. (B) Representative image of a non-inverted organoid found in suspension culture conditions. Nuclei (blue $=$ DAPI) and E-CADHERIN (red) are positioned to the basolateral side, ZO-1 (green) is expressed to the

\begin{tabular}{|c|c|}
\hline MONOLAYER CULTUREWARE & $\begin{array}{c}\text { NUMBER OF WELLS OF INTESTINAL ORGANOIDS } \\
\text { TO HARVEST (from } 50 \mu \text { dome/per well to be seeded) }\end{array}$ \\
\hline $6.5 \mathrm{~mm}$ Transwell insert & $1-2$ wells \\
\hline $12 \mathrm{~mm}$ Transwell insert & $3-4$ wells \\
\hline 6 -well plate & 6 - 8 wells \\
\hline 24-well plate & $3-4$ wells \\
\hline $96-$ well plate & $1-2$ wells \\
\hline
\end{tabular}

Table 1: Number of wells of intestinal organoids to harvest for various cultureware

\section{Discussion}

Epithelial organoid models have become powerful platforms that can be used to model tissue organization, disease progression, and identify therapeutics ${ }^{23,28,29}$. Organoid microinjection has added value to the organoids' capability of modeling infectious diseases as it allows pathogen interaction with the apical side of the host epithelium. Recent advances in microinjection techniques have optimized the injection speed in organoids and have achieved a rate of up to 90 injected apical side that faces the lumen of the organoid. (Scale bar $=$ $100 \mu \mathrm{m})$. Please click here to download this File.

\section{Supplementary Figure 3: Gene expression of} differentiated cell markers in monolayer cultures. $(A, B, C)$ ALI-differentiated monolayer culture generated in Intestinal Organoid Differentiation Medium relative to a 3D organoid culture grown with Intestinal Organoid Expansion Medium established via qPCR. Establishment of a submerged monolayer culture increases expression of each differentiated cell marker; however, differentiation as an ALI culture increases expression of each marker exponentially. Error bars $=+/$ - SEM. Please click here to download this File. Expression of MUC2, CHGA, and KRT20 in submerged and
NUMBER OF WELLS OF INTESTINAL ORGANOIDS

1 - 2 wells

$3-4$ wells
3 - 4 wells

-2 wells was preserved, and the low oxygen concentration inside the lumen allowed for the survival of obligatory-anaerobic injected bacteria $^{30}$. However, studies have noted the presence of heterogeneity in organoid populations within the same well. These differences were observed in size and shape ${ }^{31}$, expression levels of key genes ${ }^{32}$, as well as proliferation rates $^{33}$. Differential responses within the same organoid population to compounds such as forskolin and PGE2, or to 
cholera toxin, have also been described ${ }^{28,33}$. These results highlight the need for high organoid numbers in studies and limit the utilization of luminal injection.

Conventional organoid culture is based upon the encapsulation and propagation of organoids in a hydrogel. However, hydrogels can pose limitations on diffusion and introduce concentration gradients, which can increase heterogeneity ${ }^{34}$. Moreover, high variability has been documented, not only between cultures and donors but also within individual experimental conditions. Donor source, biochemical properties of the hydrogel, and intrinsic heterogeneity of the organoid as a culture system are important factors that can increase experimental variability and limit the reproducibility of results obtained in downstream applications. Both of the methods described here provide a simple means of exposing the apical side of the epithelium, allowing for the modeling of compounds and pathogens of interest by directly adding them to the culture medium. The reduction in hydrogel utilization can limit experimental variability from technical sources of error.

The apical-out intestinal organoids retain key characteristics of the organoid model system and their scalability make them more amenable to high throughput assays, compared to the 2D monolayer. However, as the organoids retain their 3D structure, the accessibility of the basal side is limited and may hinder studies requiring access to both sides simultaneously.

We have demonstrated that the polarity inversion of intestinal organoids relies on the efficient and absolute removal of ECM, while also preserving the intact structure of the organoids. Both the use of the dissociation solution to remove ECM and the anti-adherent solution to prevent organoids adherence to the plasticware contributed to ameliorating the overall efficiency of the protocol published by Co, J. Y. and colleagues $^{22}$, particularly in regard to the number of apicalout organoids produced for downstream applications.

Furthermore,we observed that our protocol supports more efficient inversion of organoids smaller than $250 \mu \mathrm{m}$ and using larger organoids may result in a reduced organoid output, due to the fragmentation caused by pipetting. Wide-bore tips, such as those indicated in the Table of Materials, may allow the utilization of larger organoids. However, wide-bore tips are less effective in ECM dissociation when compared to standard tips due to the lower applied mechanical force. Therefore, repeating steps 1.2.9-1.2.11 may be required for sufficient disruption and complete removal of all ECM remnants when working with larger organoids.

Organoids in suspension can survive for at least 2 weeks. After this period of time, we observed morphology changes and an increased number of cell death. The presence of proliferating cells in the apical-out organoids ${ }^{22}$ allows for the re-establishment of apical-in intestinal organoid cultures. This can be achieved by dissociating apical-out organoids to single cells and embedding them in ECM with Intestinal Organoid Expansion Medium.

A limitation frequently encountered in protocols describing the establishment of intestinal organoids in suspension cultures is the generation of large aggregates. This impacts several variables such as efficiency, reproducibility of the morphological features, permeability to compounds, and paracrine signaling. Similar to the protocol published by Co, J. Y. and colleagues, here we confirm to have obtained polarity inversion of at least $97 \%$ of all the suspended organoids without shearing after 3 days in suspension. However, in contrast to the publication, we have introduced a mechanical dissociation step in order to reduce the formation of large aggregates and increase yield. Since this procedure may 
damage the epithelium of the organoids, we extended the organoids incubation period for an additional 2 days to allow complete epithelium recovery and ensure high quality cultures for downstream applications. The introduction of constant agitation with the use of an incubator shaker or a spinner flask could potentially reduce fusion events, minimize fragmentation, and increase oxygenation. These alternative approaches may maintain the cultures for longer durations, reduce cell death, and allow for further differentiation of the apical-out intestinal organoids.

The establishment of an organoid-derived 2D monolayer provides several advantages and disadvantages compared to the inverted polarity organoids. The protocol described here allows for the rapid establishment of a confluent monolayer culture, typically, in less than 7 days and the option of long-term maintenance of the cultures for an extended period of time (up to 10-weeks). The protocol and the media used here also allow for the efficient differentiation of significant numbers of cells, not always found in other organoid-derived monolayer cultures ${ }^{16}$. Establishing a monolayer on a cell culture insert membrane allows for simultaneous access to both the apical and basolateral sides of the epithelium making them ideal for studies in barrier integrity and epithelial transport. This simplified access also renders them more amenable to infection and drug treatment studies. Furthermore, these cultures maintain many of the characteristics unique to the donor, maintaining their relevance for patient-specific studies. The ALI culture method also facilitates the differentiation of a more functional epithelium composed of both secretory and absorptive cell types, making it more representative of the human intestinal epithelium. The relative stability of these cultures also allows for them to be maintained for a prolonged period of time, providing the possibility for long-term studies.
However, limitations of this approach are the high number of cells required to establish a confluent monolayer and the need to maintain complete confluency to have a functional separation between the apical and basolateral chambers. The characteristic crypt architecture, which can be modeled in the $3 D$ organoid cultures is also lost upon establishing a monolayer culture. Nevertheless, the experimentally friendly format of the culture and the ease at which the apical and basolateral sides of the epithelium can be accessed, make it a powerful tool for the study of intestinal physiology.

\section{Disclosures}

G. S., W. C., and S. S. are employees of STEMCELL Technologies Ltd., Cambridge (UK). M. S., F. E., S. L., A. E., and R. K. C. are employees of STEMCELL Technologies Inc., Vancouver (Canada).

\section{Acknowledgments}

This research was funded by the Horizon 2020 grant OrganoVIR 812673 on the project Organoids for Virus Research - An innovative training-ITN programme.

\section{References}

1. Gehart, H., Clevers, H. Tales from the crypt: new insights into intestinal stem cells. Nature Reviews Gastroenterology and Hepatology. 16 (1), 19-34 (2019).

2. Schneeberger, K., Roth, S., Nieuwenhuis, E. E. S., Middendorp, S. Intestinal epithelial cell polarity defects in disease: lessons from microvillus inclusion disease. Disease Models \& Mechanisms. 11 (2) (2018).

3. Klunder, L. J., Faber, K. N., Dijkstra, G., Van ljzendoorn, S. C. D. Mechanisms of cell polarity - Controlled epithelial homeostasis and immunity in the intestine. Cold Spring Harbor Perspectives in Biology. 9 (7), a027888 (2017). 
4. DiMarco, R. L., Hunt, D. R., Dewi, R. E., Heilshorn, S. C. Improvement of paracellular transport in the Caco-2 drug screening model using protein-engineered substrates. Biomaterials. 129, 152-162 (2017).

5. Sun, H., Chow, E. C., Liu, S., Du, Y., Pang, K. S. The Caco-2 cell monolayer: usefulness and limitations. Expert Opinion on Drug Metabolism \& Toxicology. 4 (4), 395-411 (2008).

6. Clevers, H. Modeling development and disease with organoids. Cell. 165 (7), 1586-1597 (2016).

7. Dutta, D., Heo, I., Clevers, H. Disease modeling in stem cell-derived 3D organoid systems. Trends in Molecular Medicine. 23 (5), 393-410 (2017).

8. Zhang, Y.-G., Wu, S., Xia, Y., Sun, J. Salmonellainfected crypt-derived intestinal organoid culture system for host-bacterial interactions. Physiological Reports. 2 (9), e12147 (2014).

9. Nigro, G., Rossi, R., Commere, P.-H., Jay, P., Sansonetti, P. J. The cytosolic bacterial peptidoglycan sensor Nod2 affords stem cell protection and links microbes to gut epithelial regeneration. Cell Host \& Microbe. 15 (6), 792-798 (2014).

10. Heo, I. et al. Modelling Cryptosporidium infection in human small intestinal and lung organoids. Nature Microbiology. 3 (7), 814-823 (2018).

11. Bartfeld, S. et al. In vitro expansion of human gastric epithelial stem cells and their responses to bacterial infection. Gastroenterology. 148 (1), 126-136.e6 (2015).

12. Leslie, J. L. et al. Persistence and toxin production by Clostridium difficile within human intestinal organoids result in disruption of epithelial paracellular barrier function. Infection and Immunity. 83 (1), 138-145 (2015).
13. Pleguezuelos-Manzano, C. et al. Mutational signature in colorectal cancer caused by genotoxic pks(+) E. coli. Nature. 580 (7802), 269-273 (2020).

14. Fernando, E. H. et al. A simple, cost-effective method for generating murine colonic $3 \mathrm{D}$ enteroids and $2 \mathrm{D}$ monolayers for studies of primary epithelial cell function. American journal of physiology. Gastrointestinal and Liver Physiology. 313 (5), G467-G475 (2017).

15. Moon, C., VanDussen, K. L., Miyoshi, H., Stappenbeck, T. S. Development of a primary mouse intestinal epithelial cell monolayer culture system to evaluate factors that modulate IgA transcytosis. Mucosal Immunology. 7 (4), 818-828 (2014).

16. Wang, Y. et al. Long-term culture captures injury-repair cycles of colonic stem cells. Cell. 179 (5), 1144-1159.e15 (2019).

17. Noel, G. et al. A primary human macrophage-enteroid coculture model to investigate mucosal gut physiology and host-pathogen interactions. Scientific Reports. 7, 45270 (2017).

18. VanDussen, K. L. et al. Development of an enhanced human gastrointestinal epithelial culture system to facilitate patient-based assays. Gut. 64 (6), 911-920 (2015).

19. Thorne, C. A. et al. Enteroid monolayers reveal an autonomous WNT and BMP circuit controlling intestinal epithelial growth and organization. Developmental Cell. 44 (5), 624-633.e4 (2018).

20. Wang, A. Z., Ojakian, G. K., Nelson, W. J. Steps in the morphogenesis of a polarized epithelium: I. Uncoupling the roles of cell-cell and cell-substratum contact in establishing plasma membrane polarity in multicellular 
epithelial (MDCK) cysts. Journal of Cell Science. 95 (1), 137-151 (1990).

21. Wang, A. Z., Ojakian, G. K., Nelson, W. J. Steps in the morphogenesis of a polarized epithelium: II. Disassembly and assembly of plasma membrane domains during reversal of epithelial cell polarity in multicellular epithelial (MDCK) cysts. Journal of Cell Science. 95 (1), 153-165 (1990).

22. Co, J. Y. et al. Controlling epithelial polarity: a human enteroid model for host-pathogen interactions. Cell Reports. 26 (9), 2509-2520.e4 (2019).

23. Sato, T. et al. Long-term expansion of epithelial organoids from human colon, adenoma, adenocarcinoma, and Barrett's epithelium. Gastroenterology. 141 (5), 1762-1772 (2011).

24. Dodt, H.-U. et al. Ultramicroscopy: three-dimensional visualization of neuronal networks in the whole mouse brain. Nature Methods. 4 (4), 331-336 (2007).

25. Fujii, M. et al. Human intestinal organoids maintain selfrenewal capacity and cellular diversity in niche-inspired culture condition. Cell Stem Cell. 23 (6), 787-793.e6 (2018).

26. Crowley, S. M. et al. Intestinal restriction of Salmonella Typhimurium requires caspase-1 and caspase-11 epithelial intrinsic inflammasomes. PLoS Pathogens. 16 (4), e1008498 (2020).

27. Rees, W. D. et al. Enteroids derived from inflammatory bowel disease patients display dysregulated endoplasmic reticulum stress pathways, leading to differential inflammatory responses and dendritic cell maturation. Journal of Crohn's \& Colitis. 14 (7), 948-961 (2020).
28. Dekkers, J. F. et al. A functional CFTR assay using primary cystic fibrosis intestinal organoids. Nature Medicine. 19 (7), 939-945 (2013).

29. Almeqdadi, M., Mana, M. D., Roper, J., Yilmaz, Ö. H. Gut organoids: mini-tissues in culture to study intestinal physiology and disease. American Journal of Physiology. Cell Physiology. 317 (3), C405-C419 (2019).

30. Williamson, I. A. et al. A high-throughput organoid microinjection platform to study gastrointestinal microbiota and luminal physiology. Cellular and Molecular Gastroenterology and Hepatology. 6 (3), 301-319 (2018).

31. Kassis, T., Hernandez-Gordillo, V., Langer, R., Griffith, L. G. OrgaQuant: human intestinal organoid localization and quantification using deep convolutional neural networks. Scientific Reports. 9 (1), 12479 (2019).

32. Gracz, A. D. et al. A high-throughput platform for stem cell niche co-cultures and downstream gene expression analysis. Nature Cell Biology. 17 (3), 340-349 (2015).

33. Gunasekara, D. B. et al. Development of arrayed colonic organoids for screening of secretagogues associated with enterotoxins. Analytical Chemistry. 90 (3), 1941-1950 (2018).

34. Shin, W. et al. Spatiotemporal gradient and instability of Wnt induce heterogeneous growth and differentiation of human intestinal organoids. iScience. 23 (8), 101372 (2020). 